\title{
Ophthalmologic approach to babies with cerebral visual impairment
}

\author{
Şefay Aysun İdil ${ }^{1,2 \oplus}$, Deniz Altınbay ${ }^{2 \oplus}$, Esra Şahl1 ${ }^{1,2 \oplus}$, Pınar Bingöl Kızıltunçç, \\ Hatice Semrin Timlioğlu İper ${ }^{20}$, Kadriye Erkan Turann ${ }^{20}$, \\ Damla Ergintürk Acar ${ }^{2}$, Fatma Merve Bektaş ${ }^{2}$ \\ ${ }^{1}$ Department of Ophthalmology and ${ }^{2}$ Center of Vision Research and Low Vision Rehabilitation, Ankara University Faculty of Medicine, \\ Ankara, Turkey.
}

\begin{abstract}
Background. Cerebral visual impairment (CVI) is an increasingly common type of visual disturbance in infants and children. The increased incidence is associated with improved neonatal intensive care services and survival of premature infants, especially in developed countries and our country. For accurate ophthalmological evaluation, it is important to know the behavioral responses to visual stimuli that are unique to CVI such as color preference, need for movement, visual latency, visual field preference, and difficulty in visual complexity.

Methods. This review is prepared to draw attention to the subject within the scope of Ankara University Vision, Artificial Vision and Low Vision Rehabilitation training programs.

Results. The most common causes are hypoxia and perinatal ischemia. Ocular structures are generally normal or are not sufficient to explain the visual impairment. Diagnosis and habilitation methods differ from visual impairment of ocular origin. As a result, early diagnosis of CVI in infants and toddlers and an effective visual habilitation with a multidisciplinary approach where ophthalmologists and pediatricians lead the team is very valuable.

Conclusions. Early diagnosis and early visual habilitation will increase the quality of life of babies and will provide important gains for families and therefore the whole society. Pediatricians should be familiar with this group of disturbances and the available resources, as they are best placed to refer the child for evaluation and rehabilitation and encourage the family for follow-up.
\end{abstract}

Key words: visual impairment, cerebral, low vision, visual habilitation.

Visual impairments in infants and toddlers can be seen due to ocular and cerebral causes or both. Cerebral visual impairment (CVI) is a disorder that we have encountered more commonly in infants and toddlers, recently. This situation is closely related to the increase in neonatal care services and keeping the infants alive that were previously lost in the early period, especially in developed countries and

$凶$ Şefay Aysun İdil

sefayaysunidil@gmail.com

Received 24th June 2020, revised 19th July 2020, accepted 22nd July 2020. our country. In addition, many factors such as scientific developments in infertility treatment, increased preterm births, multiple gestations and ongoing high consanguineous marriage rates affect the frequency of CVI. The frequency of CVI differs in developed and developing countries. In the United States, CVI was found in $2.4 \%$ of 7200 children who applied to the pediatric ophthalmology clinic $^{1}$, while it was present in $19 \%$ of children with severe visual impairment. ${ }^{2}$ In the U.K., $40-48 \%$ of children under 15 years of age with visual impairment had CVI.,4 In New Zealand, 30\% of childhood blindness is reported to be associated with CVI, 
and the calculated prevalence was $0.02 \% .^{5}$ In India, isolated CVI was found in 33\% of children with low vision, and both cerebral and ocular pathologies were detected in $11 \% .^{6}$ Among children with severe visual impairment, CVI prevalence was reported as $2.5 \%$ in Malaysia and $0.3 \%$ in China. ${ }^{7,8}$

To date, this condition has been expressed with definitions such as cortical blindness, cortical visual impairment, central blindness, neurological visual impairment, and cerebral visual impairment. Since the involvement is not limited to the cortex, it is preferable to use the word 'cerebral' instead of cortical. Since the vision can be improved in this age group and even only the presence of light perception is considered as a developmental emergency, it is preferable to use 'visual impairment' instead of blindness.

Vision is a complex system, only part of which is created by the eye. The primary visual center in the brain is the occipital cortex (striate cortex). However, the extrastriate cortexrelated pathways of the brain are required for the cognitive visual function. With the dorsal stream that connects the visual cortex to the parietal lobes, movement function (the 'where' stream); with the ventral stream that connects the visual cortex to the temporal lobes, recognition function (the 'what' stream) is performed. Frontal brain areas perform the task of managing by using this complex tool. ${ }^{9,10}$

All babies learn to see after they are born. In order to learn to see, adequate visual stimulation is required in addition to healthy eyes, visual pathways, and related centers in the brain. Brain plasticity is extremely important for gaining vision in infants and toddlers. Although plasticity continues until the age of ten, the best time for proper stimulation is the early infancy period. Therefore, it is vital to start visual habilitation early. ${ }^{11,12}$ Diagnosis and rehabilitation of CVI differ from visual impairments caused by other reasons..$^{13,14}$

The causes of CVI are congenital or acquired (Table I). ${ }^{15-18}$ The most common cause is perinatal hypoxia and ischemia. Neurological and ophthalmological deficits are seen together in most of the children with CVI. Frequent neurological deficits are seizures (requiring anticonvulsant therapy), cerebral palsy, hemiparesis, hydrocephalus, hypotonia, hearing loss, microcephaly, and learning difficulties. ${ }^{1}$ In addition, disorders that may present with symptoms and findings similar to those of CVI, such as autism spectrum disorders, developmental coordination disorders, delayed visual maturation, learning difficulties, bilateral central scotoma, dyskinetic eye movement disorders, and mental retardation, should be kept in mind in the differential diagnosis. ${ }^{19,20}$

InCVI, ocularstructures are generally completely normal, or the pathologies are not sufficient to explain visual loss. In these patients, some parts of the brain, such as the visual cortex or visual pathways, are damaged, and abnormal findings can be detected by imaging methods. However, CVI may be accompanied by refractive errors, strabismus, ocular motor apraxia, and optic atrophy. Nystagmus is rare and should alert the physician in terms of anterior visual pathway damage. Optical atrophy can be seen in the case of severe hypoxia and ischemia and its presence should alert the physician in terms of the severity of the condition and poorness of the prognosis.

\section{OPHTHALMOLOGICAL EVALUATION IN CVI}

Appropriate tests and methods should be used regarding the age and underlying pathology. Behavioral responses against visual stimuli specific to CVI are of great importance. Detailed anamnesis should be taken with the company of the family or child's caregiver and observations of the family about the child's behavior should be evaluated. To avoid stimulating other senses, the examination room should be quiet and dull, the physician performing the examination should be dressed in a plain solid color if possible, and avoid stimuli such as odors. The fixation object/ toy should be selected according to the child's 
Table I. Causes of cerebral visual impairment.

Congenital and Perinatal Causes

Asphyxia and perinatal hypoxic encephalopathy

Placenta previa, abruptio placentae, uterine rupture, umbilical cord prolapse, severe shoulder dystocia, maternal diabetes, maternal infection, rapid labor

Intraventricular hemorrhage

Post-hemorrhagic hydrocephalus

Hydrocephalus-shunt malfunction

Periventricular leukomalacia

Post-natal hypoxia-ischemia

Neonatal stroke

Cerebrovascular problems, cerebral artery occlusion

Prematurity

Structural anomalies

Occipital or parietal encephalocele, meningomyelocele (spina bifida), Dandy-Walker syndrome, primary microcephaly, schizencephaly, primary congenital hydrocephalus, polymicrogria, corpus callosum agenesis

Neurological diseases

Cerebral palsy

Infections

TORCH infections

Multiple gestation

Premature birth, periventricular leukomalacia

Hereditary diseases

HANAC syndrome, Aicardi-Goutieres syndrome,

Walker-Warburg syndrome, MELAS syndrome,

ornithine transcarbamylase deficiency, Fabry disease,

X-linked adrenoleukodystrophy

Acquired Causes

Trauma, accident

Metabolic and neurodegenerative conditions

Hypoglycemia, kernicterus, carbon monoxide intoxication, uremia, hypovolemia

Hypoxia

Infections Sepsis, encephalitis, meningitis

Tumors

Toxic causes

Vincristine, cyclosporine etc.

age and interest (Fig. 1a, 1b). Cerebral pathology leading to visual impairment, other sensory and neurological deficits that may affect the child's expected visual behavior and response, should be considered.

Evaluations made during the detailed ophthalmological examination;

a. Binocular vision: Although it is difficult to assess binocular vision in these children, it is considered to be defective. Strabismus in infants with CVI, disrupts the formation of the fusion mechanisms and prevents binocularity, and development of depth perception, and three-dimensional vision. ${ }^{21,22}$ b. Oculomotor evaluation: Before starting the examination, it should be observed whether the child has a head position. Binocular and monocular eye movements should be evaluated. In assessing the ocular misalignment, it is appropriate to use tests evaluating the corneal light reflex, such as Hirschberg and Krimsky tests, due to the lack of cooperation in these patients. The presence of optokinetic nystagmus response is evaluated by rotating the optokinetic drum, a tool with alternating black and white vertical stripes, in front of the patient. The response consists of slow eye movements in the direction of the stimulus, followed by fast corrective eye movements, indicates an intact visual pathway. 

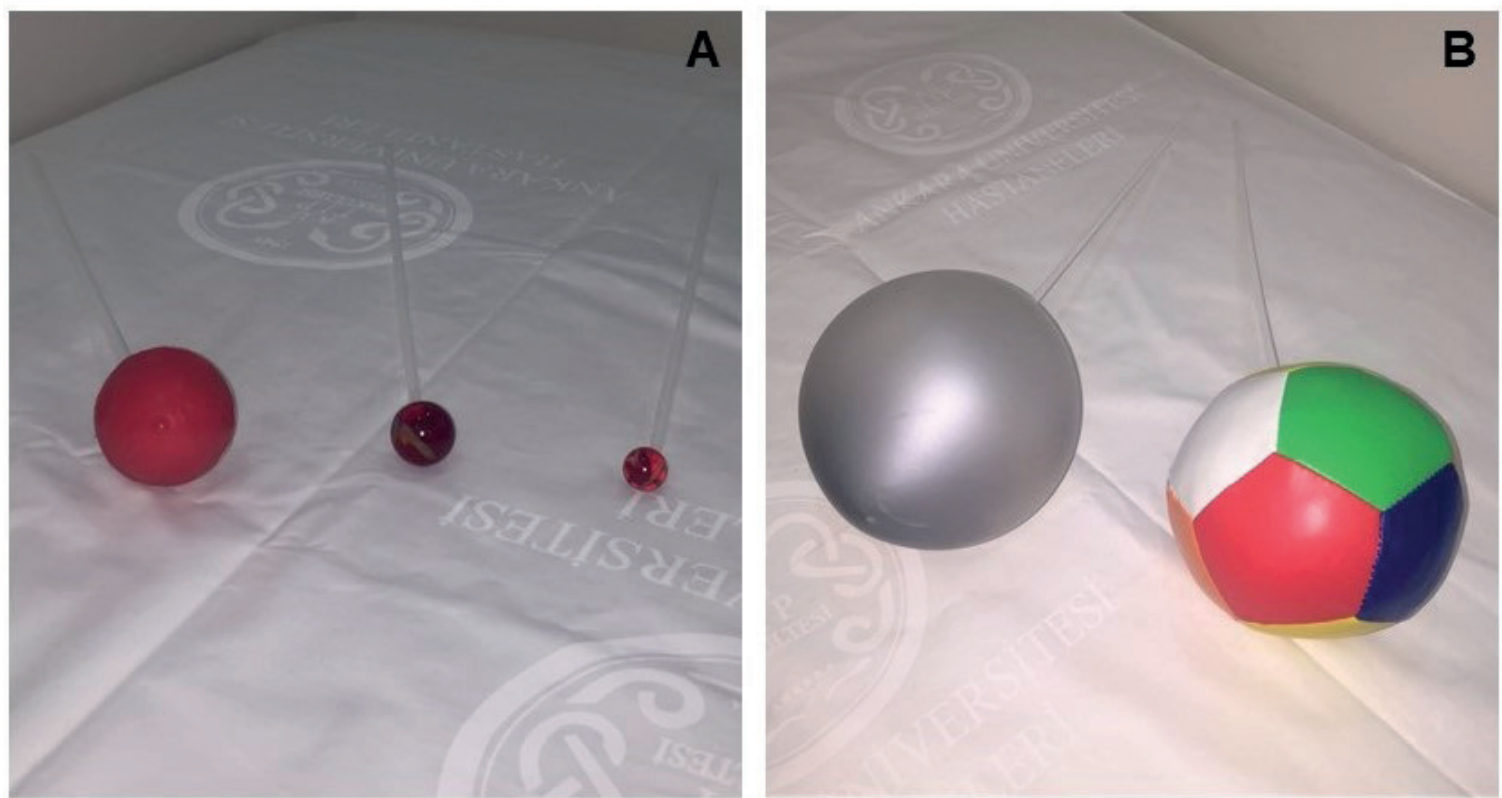

Fig. 1. Red objects with a diameter of $1 \mathrm{~cm}, 2.5 \mathrm{~cm}$ and $5 \mathrm{~cm}(1 \mathrm{a})$, gray and colored objects with a diameter of $25 \mathrm{~cm}(1 \mathrm{~b})$.

c. Visual acuity: Preferential-looking tests, based on observing the child's behavior when encountering different visual stimuli, and visual evoked potentials (VEP) are reliable and valid methods used frequently. In preferentiallooking test, the child who is presented with two stimulus areas, one with stripes and the other with a homogeneous gray area, is expected to prefer to look at the striped one. Although VEP provides vision measurement without the influence of motor ability, behavioral vision measurements are more useful in defining the function. ${ }^{23}$

d. Contrast sensitivity: Contrast sensitivity is an important function that affects daily activities such as communication, access to information, orientation and mobility skills. Besides many factors related to the object, environment, and eyes, contrast sensitivity is expected to be mainly affected in CVI. ${ }^{24}$

e. Color vision: In most children with CVI color vision is normal and color selection is preserved. All color vision tests should be carried out in daylight. f. Visual Field: Visual fields are examined with a method suitable for the child's age and cooperation. The confrontation method should be preferred between 4 months- 6 years of age and automated visual field tests, for older children. The confrontation method, which is applied by the physician moving the object slowly from the periphery to the center in the nasal, upper, temporal, and lower quadrant, respectively, is the most widely used. ${ }^{25,26}$

\section{g. Electrophysiological methods:}

Electrophysiological tests detect neurological and concomitant ocular pathologies and may determine the prognosis of CVI. For this purpose, VEP tests (pattern, flash, sweep) have gained priority in CVI. ${ }^{19}$ However, the interpretation of pattern and flash VEP results in young children is difficult and debatable. ${ }^{23,27}$ Sweep VEP test is more suitable for quantitative measurement. Although thought to define visual acuity correctly, limitations such as concomitant neurological disorders, general status of health, medications make it difficult to acquire an exact match..$^{28,29}$ 


\section{BEHAVIORAL RESPONSES TO VISUAL STIMULI IN CVI}

The presence of behavioral responses can vary depending on the region and the severity of the cerebral pathology. Besides, factors pertaining to the child (fatigue, post-seizure period, overstimulation), the target (color, complexity) and the environment (simple, crowded) may affect these behavioral responses and may differ from the child's home where he/she feels more comfortable. All conditions in the examination must be recorded.

If these behavioral answers are summarized;

a. Color preference: Color vision is close to normal for most children with CVI because color is represented bilaterally in the visual cortex and these areas are generally preserved. Children with CVI are more interested in visual targets with distinct colors. Most prefer red and yellow. However, having a red/yellow color preference does not lead to the diagnosis of CVI, or any other color selection does not exclude CVI diagnosis. ${ }^{30}$

If the child has a specific color preference, it is important to use this color during the child's habilitation. Attaching an object with the preferred color to the spoon or the feeding bottle while eating can increase the motivation of the child, increase the vision, and improve visual learning. ${ }^{31}$ Children with CVI first see one-color objects. As the severity of the disease decreases, they begin to see more colors and patterns, and color and pattern preferences do not remain as the CVI improves. ${ }^{30}$

b. Requirement for Movement: The majority of children with CVI tend to turn towards moving objects, or react differently to these objects, such as smiling or remaining silent. ${ }^{32}$ These children usually like to watch the traffic from the window or watch television for a long time.

Perception of movement in children with CVI differs according to the damaged area of the brain. The pathways mentioned above are necessary for cognitive visual functions. ${ }^{33}$ The occipitoparietal area detects motion and damage to the posterior periventricular white matter may alter perception of motion. ${ }^{34}$ This type of lesions are often due to prematurity; these babies have problems detecting fast-moving objects and prefer slower moving objects. ${ }^{10} \mathrm{In}$ children with damage is mainly in the occipital lobe and not in the occipitoparietal pathway, perception of movement is normal but visual impairment is present. ${ }^{35}$ These children try to create the feeling of seeing with head shaking, or turning around themselves.

Habilitation may therefore make use of moving objects to initiate and maintain seeing. As the severity of CVI decreases, moving is only needed when the infant is distracted, and may even be left out with further improvement.

c. Visual latency: Visual latency is the time between looking at a target and seeing the target. Visual responses are slow and delayed in children with CVI. They can ignore the target at first. After enough time, they can focus on the target and turn towards the target. The presence and duration of visual latency varies from one child to the other. ${ }^{36}$

With the improvement of CVI, latency is shortened, and a delay may occur only when the child is tired, stressed, or overstimulated. With full recovery, the latency may be eliminated completely.

d. Visual field preference: Most of these children have a visual field preference that varies depending on the brain's damaged area. ${ }^{30,37}$ There is a dorsal stream dysfunction in hypoxic-ischemic encephalopathy and periventricular leukomalacia. Especially in periventricular leukomalacia, the lateral ventricles are enlarged and the upper fibers of the optical radiation are affected: this creates defect in binocular lower visual field as the most frequent finding 38,39 The right or left visual field and rarely, a central visual field may be preferred. ${ }^{30}$ The visual field preference may not be available in approximately $30 \%$ of cases. 
These children often prefer either the right or left visual field at the initial stage of the disease (Fig. 2). At this stage, training should be started by showing the toy from the preferred visual field. As the severity of CVI decreases, these children start using both the right and left visual fields, but still, one is dominant. Significant improvement results in the disappearance of any preferences for visual fields.

\section{e. Difficulties discriminating or interpreting} complex visual patterns: This is one of the most prominent, long lasting, and resistant characteristics. It may persist for a lifetime to some degree. These children need support in academic life. ${ }^{30,40}$ The problem is thought to be related to the damage of the dorsal stream and

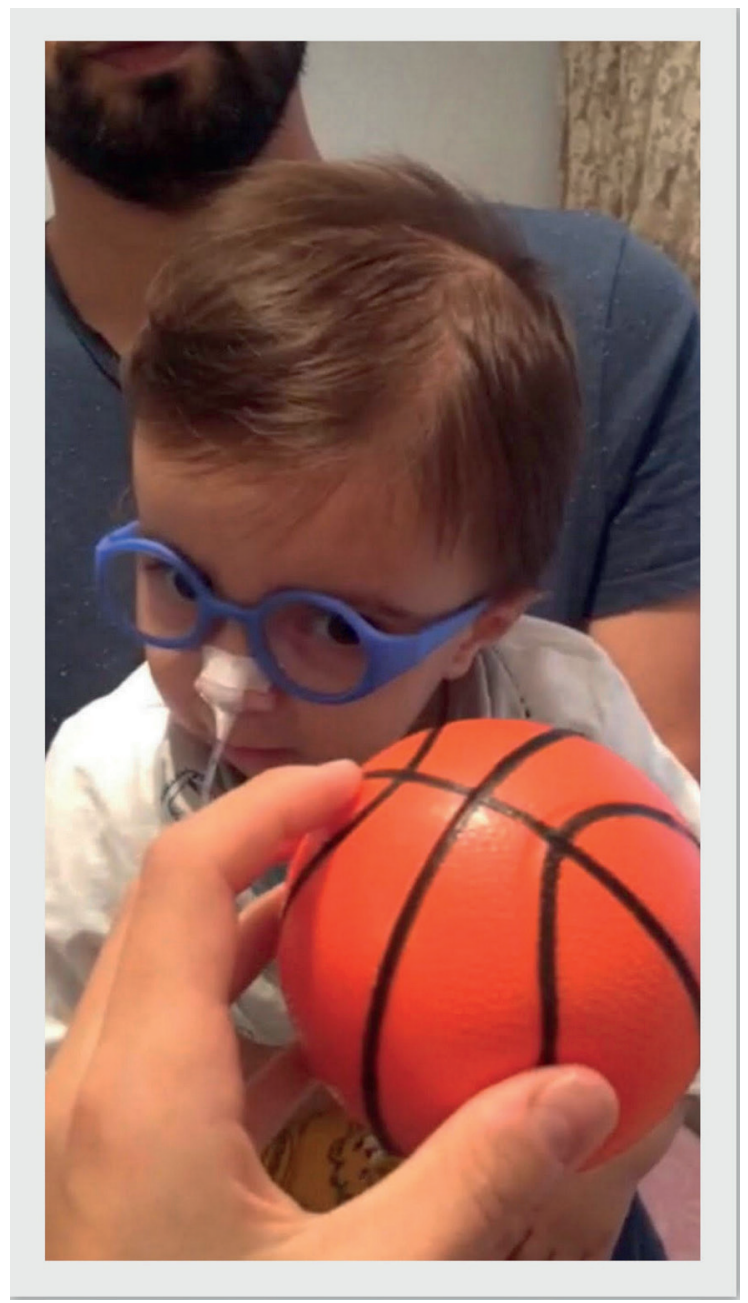

Fig. 2. A child with a diagnosis of cerebral visual impairment and left visual field preference. theintersected areas of the occipital and temporal lobes which serve as the 'image library'. ${ }^{41}$ Difficulties discriminating or interpreting complex visual patterns can be related to objects (multicolor objects), sequences (multiple toys), the environment (crowded environments), as well as sensations (simultaneous stimulation of multiple senses). Patients seem to be restless in crowded and varied environmental conditions, or prefer to sleep to avoid complexity. These children initially prefer to look at a single object. The next step would include showing a second toy or a multi-color sample of the one-color toy. As the disease recovers, the child can see objects ranging from simple to complex. The visual activity can be improved from fully controlled environments to complex environments including other stimuli. ${ }^{30,42}$

\section{f. Light-gazing and non-purposeful gaze:}

Staring at the light is an impulsive behavior. Parents define this behavior by saying that their children are looking at the light that leaks from the window or the lamp on the ceiling for a long time, but not the target. ${ }^{30}$ The non-purposeful gaze is defined as the child not being able to look at the target shown or focusing when there is no target. ${ }^{30}$ While looking at the light is normal in newborn babies, the continuation of this behavior in children with CVI signs, indicates that visual development is delayed. ${ }^{30}$ In the classroom, instead of looking at the blackboard, they may aimlessly stare at the light coming from the window, or turn their heads to the other side and look as if they are not interested. The purpose of this action is to try to accomplish perception by using senses other than vision. ${ }^{43}$

The behavior of looking at the light was observed in $60 \%$ of children with CVI. In computed brain tomography findings in children with or without his behavior, no definite neuro-anatomical difference could be demonstrated..$^{44}$ The behavior of looking at the light and non-purposeful gaze is not related to the severity and etiology of the disease and visual level. When there is even a slight impact on the striate cortex, not causing a significant 
decrease in vision, may result in this behavior. The intensity of light-gazing decreases as CVI improves. ${ }^{37}$

g. Difficulty with distance viewing: As difficulties discriminating or interpreting complex visual patterns, this is also thought to be related to the dorsal stream. Therefore, problems with complex visual patterns may be associated with those in distance viewing. ${ }^{10,41,42}$ The latter is one of the resistant features of CVI. The possibility of viewing distant objects increases with the simplicity of the environment. Initially, he/she can only look at objects at a distance of $40-45 \mathrm{~cm}$, but as the disease improves, the distance $\mathrm{s} /$ he can see increases, but nevertheless, it will still be difficult to see in a complex environment.

h. Atypical visual reflexes: Most children with CVI respond atypically to two reflexes, called blink reflexes and threat responses, which are expected to exist from birth. Normally, when a child's nasal bridge is tapped gently, s/he blinks simultaneously. The threat response is the blink when a hand is moved quickly in the midline of the baby's face. In most of these children, these reflexes are either absent or delayed. In the recovery process, a delayed blink reflex may appear first, and then starts to gain continuity; the response to threat is observed intermittently. In the advanced phase of recovery, both reflexes are constantly present.

i. Difficulty in visual innovations: Since they have difficulties to process the information they see these children prefer familiar objects that can be easily remembered..$^{30}$ The same object is offered repeatedly during training. The child is then introduced to a new object with similar properties. Initially, s/he shows interest only in the familiar objects, and with recovery, new objects that have common features with the familiar ones. In the case of improvement, there is no limitation in object preference. ${ }^{45}$

j. Visually guided reach: Reaching and catching a target are motor behaviors, but they are also based on the dorsal and ventral stream of the visual system. Ventral stream functions enable to us to see and recognize the target while dorsal stream functions allow us to reach and take it. Therefore, achieving a target is a multistep process taking place within the brain. ${ }^{46,47}$ Any impairment in this complex system may affect ordinary movements. In children with CVI, seeing and reaching a target cannot be performed simultaneously. Characteristics of the target and the environment also affect the reaching function. ${ }^{30}$ During these evaluations the child's motor functions should also take into account.

With the improvement of CVI, firstly, reaching to familiar and preferred color objects on a plain background is seen. Then, looking and reaching may be done in quick succession. With full recovery, looking and reaching appear simultaneously. ${ }^{30}$

\section{Habilitation}

Except for hydrocephalus and metabolic diseases, there is no etiological treatment for CVI. The presence of multiple disabilities can adversely affect the habilitation. The crucial point is to ensure the communication and interaction of the child with the external world and to increase participation in daily life. Various clinical studies have been conducted to improve the quality of life of children with CVI, but the improvement of visual functions has not been sufficiently emphasized. ${ }^{48,49}$

Vision re/habilitation therapy can be used for both ocular and cerebral visual impairment. However, rehabilitation strategies vary depending on the underlying cause. The strategies used in ocular low vision will not be effective in CVI. In ocular problems, the desired results may not be achieved with rehabilitation. However, in CVI where an ophthalmological examination is usually normal, vision is more likely to improve. ${ }^{50}$ Groenveld et al., ${ }^{14}$ stated that children with CVI always tend to recover and there is always some residual vision. Infants with brain damage may benefit from appropriate stimulation and experience an 
increase in vision. ${ }^{11}$ This was explained by brain plasticity, a concept first mentioned by Hubel and Wiesel in 1981 and it made them win the Nobel Prize. ${ }^{51}$ In CVI, stimulation for visual development should be applied within the crucial time window for visual development, or the 'critical period'.

In summary, CVI in infants and toddlers can benefit from early diagnosis and effective visual habilitation by a multidisciplinary team where ophthalmologists and pediatricians are collaborating.

\section{REFERENCES}

1. Huo R, Burden SK, Hoyt CS, Good WV. Chronic cortical visual impairment in children: aetiology, prognosis, and associated neurological deficits. Br J Ophthalmol 1999; 83: 670-675.

2. Steinkuller PG, Du L, Gilbert C, Foster A, Collins ML, Coats DK. Childhood blindness. J AAPOS 1999; 3: 26-32.

3. Rahi JS, Cable N, British Childhood Visual Impairment Study Group.Severe visual impairment and blindness in children in the UK. Lancet 2003; 362: 1359-1365.

4. Rahi JS. Childhood blindness: a UK epidemiological perspective. Eye (Lond) 2007; 21: 1249-1253.

5. Chong C, Dai S. Cross-sectional study on childhood cerebral visual impairment in New Zealand. J AAPOS 2014; 18: 71-74.

6. Pehere NK, Narasaiah A, Dutton GN. Cerebral visual impairment is a major cause of profound visual impairment in children aged less than 3 years: a study from tertiary eye care center in South India. Indian J Ophthalmol 2019; 67: 1544-1547.

7. Patel DK, Tajunisah I, Gilbert C, Subrayan V. Childhood blindness and severe visual impairment in Malaysia: a nationwide study. Eye (Lond) 2011; 25: 436-442.

8. Hornby SJ, Xiao Y, Gilbert CE, et al. Causes of childhood blindness in the People's Republic of China: results from 1131 blind school students in 18 provinces. Br J Ophthalmol 1999; 83: 929-932.

9. Cooper SA, O'Sullivan M. Here, there and everywhere: higher visual function and the dorsal visual stream. Pract Neurol 2016; 16:176-183.

10. Philip SS, Dutton GN. Identifying and characterising cerebral visual impairment in children: a review. Clin Exp Optom 2014; 97: 196-208.
11. Roman-Lantzy C. Cortical visual impairment: an overview. In: Cortical Visual Impairment: An Approach to Assessment and Intervention. New York, NY: AFB Press, 2007: 5-11.

12. Tychsen L. Critical periods for development of visual acuity, dept perception, and eye tracking. In: Bailey DB Jr, Brure JT, Symons FJ, Lichtman JW (eds). Critical Thinking About Critical Periods. A Series from the National Center for Early Development and Learning. Baltimore, MD: Paul H. Brookes Publishing, 2001: 66-80.

13. Martín MB, Santos-Lozano A, Martín-Hernández J, et al. Cerebral versus Ocular Visual Impairment: The Impact on Developmental Neuroplasticity. Front Psychol 2016; 7: 1958.

14. Groenveld M, Jan JE, Leader P. Observations on the habilitation of children with cortical visual impairment. J Vis Impair Blind 1990; 84: 11-15.

15. Brodsky MC, Fray KJ, Glasier CM. Perinatal cortical and subcortical visual loss: mechanisms of injury and associated ophthalmologic signs. Ophthalmology 2002; 109: 85-94.

16. Hoyt CS. Visual function in the brain-damaged child. Eye (Lond) 2003; 17: 369-384.

17. Brodsky MC. The Apparently Blind Infant. In: Chapter 1 The Apparently Blind Infant. Pediatric Neuro-Ophthalmology (2nd ed). New York: Springer, 2010: 1-58.

18. Ozturk T, Er D, Yaman A, Berk AT. Changing trends over the last decade in the aetiology of childhood blindness: a study from a tertiary referral centre. Br J Ophthalmol 2016; 100: 166-171.

19. Chang MY, Borchert MS. Advances in the evaluation and management of cortical/cerebral visual impairment in children. Surv Ophthalmol 2020; 65: 708-724.

20. Lueck AH, Dutton GN, Chokron S. Profiling children with cerebral visual impairment using multiple methods of assessment to aid in differential diagnosis. Semin Pediatr Neurol 2019; 31: 5-14.

21. O'Connor AR, Birch EE, Spencer R. Factors affecting development of motor skills in extremely low birth weight children. Strabismus 2009; 17: 20-23.

22. Ortibus EL, De Cock PP, Lagae LG. Visual perception in preterm children: what are we currently measuring? Pediatr Neurol 2011; 45: 1-10.

23. Watson T, Orel-Bixler D, Haegerstrom-Portnoy G. Early visual-evoked potential acuity and future behavioral acuity in cortical visual impairment. Optom Vis Sci 2010; 87: 80-86. 
24. Good WV, Hou C, Norcia AM. Spatial contrast sensitivity vision loss in children with cortical visual impairment. Invest Ophthalmol Vis Sci 2012; 53: 7730-7734.

25. Porro GL. A new behavioural visual field test for clinical use in pediatric neuro-ophthalmology. Neuroophthalmol 1998; 19: 205-214.

26. Koenraads $\mathrm{Y}$, Braun KP, van der Linden DC, Imhof SM, Porro GL. Perimetry in young and neurologically impaired children: the Behavioral Visual Field (BEFIE) Screening Test revisited. JAMA Ophthalmol 2015; 133: 319-325.

27. Wong VC. Cortical blindness in children: a study of etiology and prognosis. Pediatr Neurol 1991; 7: 178185.

28. Skoczenski AM, Good WV. Vernier acuity is selectively affected in infants and children with cortical visual impairment. Dev Med Child Neurol 2004; 46: 526-532.

29. Good WV. Development of a quantitative method to measure vision in children with chronic cortical visual impairment. Trans Am Ophthalmol Soc 2001; 99: 253-269.

30. Roman-Lantzy C. Visual and Behavioral Characteristics of Children with Cortical Visual Impairment. In: Cortical Visual Impairment: An Approach to Assessment and Intervention. New York, NY: AFB Press, 2007: 20-30.

31. Cohen-Maitre SA, Haerich P. Visual attention to movement and color in children with cortical visual impairment. J Vis Impair Blind 2005; 99: 1-20.

32. Merabet LB, Mayer DL, Bauer CM, Wright D, Kran BS. Disentangling how the brain is "wired" in cortical (cerebral) visual impairment. Semin Pediatr Neurol 2017; 24: 83-91.

33. Dutton GN, McKillop EC, Saidkasimova S. Visual problems as a result of brain damage in children. $\mathrm{Br}$ J Ophthalmol 2006; 90: 932-933.

34. Pavlova M, Staudt M, Sokolov A, Birbaumer N, Krageloh-Mann I. Perception and production of biological movement in patients with early periventricular brain lesions. Brain 2003; 126(Pt 3): 692-701.

35. Goodale MA, Milner AD. Separate visual pathways for perception and action. Trends Neurosci 1992; 15: 20-25.

36. Pehere NK, Jacob N. Understanding low functioning cerebral visual impairment: an Indian context. Indian J Ophthalmol 2019; 67: 1536-1543.

37. Jan JE, Groenveld M. Visual behaviors and adaptations associated with cortical and ocular impairment in children. J Vis Impair Blind 1993; 87: 101-105.
38. Dutton GN, Saaed A, Fahad B, et al. Association of binocular lower visual field impairment, impaired simultaneous perception, disordered visually guided motion and inaccurate saccades in children with cerebral visual dysfunction a retrospective observational study. Eye 2004; 18: 27-34.

39. Merabet LB, Devaney KJ, Bauer CM, Panja A, Heidary G, Somers DC. Characterizing visual field deficits in cerebral/cortical visual impairment (CVI) using combined diffusion based imaging and functional retinotopic mapping: a case study. Front Syst Neurosci 2016; 10: 13.

40. Sakki HEA, Dale NJ, Sargent J, Perez-Roche T, Bowman R. Is there consensus in defining childhood cerebral visual impairment? A systematic review of terminology and definitions. Br J Ophthalmol 2018; 102: $424-432$.

41. Dutton GN. Dorsal stream dysfunction and dorsal stream dysfunction plus: a potential classification for perceptual visual impairment in the context of cerebral visual impairment? Dev Med Child Neurol 2009; 51: 170-172.

42. Lueck AH. Cortical or cerebral visual impairment in children: a brief overview. J Vis Impair Blind 2010; 104: 585-592.

43. Dutton GN, Macdonald E, Drummond SR, Saidkasimova S, Mitchell K. Clinical Features of Perceptual and Cognitive Visual Impairment in Children with Brain Damage of Early Onset. In: Dutton GN, Bax M (eds). Visual Impairment in Children Due To Damage To The Brain: Clinics in Developmental Medicine. London: John Wiley \& Sons, 2010: 106-116.

44. Jan JE, Groenveld M, Sykanda AM. Light-gazing by visually impaired children. Dev Med Child Neurol 1990; 32: 755-759.

45. Roman-Lantzy C. Functional Vision Assessment: The CVI Range. In: Cortical Visual Impairment: An Approach to Assessment and Intervention. New York, NY: AFB Press, 2007: 50-113.

46. Machado S, Cunha M, Portella CE, et al. Integration of cortical areas during performance of a catching ball task. Neurosci Lett 2008; 446: 7-10.

47. Chokron S, Dutton GN. Impact of cerebral visual impairments on motor skills: implications for developmental coordination disorders. Front Psychol 2016; 7: 1471.

48. Ellenberg JH, Nelson KB. The association of cerebral palsy with birth asphyxia: a definitional quagmire. Dev Med Child Neurol 2013; 55: 210-216.

49. van den Bos GA, Triemstra AH. Quality of life as an instrument for need assessment and outcome assessment of health care in chronic patients. Qual Health Care 1999; 8: 247-252. 
50. Roman-Lantzy C. Program Planning and Intervention. In: Cortical Visual Impairment: An Approach to Assessment and Intervention. New York, NY: AFB Press, 2007: 113-173.
51. Berlucchi G. Revisiting the 1981 Nobel Prize to Roger Sperry, David Hubel, and Torsten Wiesel on the occasion of the centennial of the Prize to Golgi and Cajal. J Hist Neurosci 2006; 15: 369-375. 